\title{
Dynamic two-axis curvature measurement using multicore fiber Bragg gratings interrogated by arrayed waveguide gratings
}

\author{
Amanda Fender, Euan J. Rigg, Robert R. J. Maier, William N. MacPherson, \\ James S. Barton, Andrew J. Moore, Julian D. C. Jones, Donghui Zhao, \\ Lin Zhang, Ian Bennion, Scott McCulloch, and Ben J. S. Jones
}

\begin{abstract}
We describe the use of arrayed waveguide gratings (AWGs) in the interrogation of fiber Bragg gratings (FBGs) for dynamic strain measurement. The ratiometric AWG output was calibrated in a static deflection experiment over a $\pm 200 \mu \varepsilon$ range. Dynamic strain measurement was demonstrated with a FBG in a conventional single-mode fiber mounted on the surface of a vibrating cantilever and on a piezoelectric actuator, giving a resolution of $0.5 \mu \varepsilon$ at $2.4 \mathrm{kHz}$. We present results of this technique extended to measure the dynamic differential strain between two FBG pairs within a multicore fiber. An arbitrary cantilever oscillation of the multicore fiber was determined from curvature measurements in two orthogonal axes at $1125 \mathrm{~Hz}$ with a resolution of $0.05 \mathrm{~m}^{-1}$. () 2006 Optical Society of America
\end{abstract}

OCIS codes: $\quad 060.2370,230.1480,120.7280$.

\section{Introduction}

In order to monitor the shape or configuration of flexible components and surfaces it is an advantage to be able to measure local curvature. There are two broad classes of optical techniques that can be employed to this end: determination of surface shape or position using external illumination, and in situ measurement using optical fibers to measure bending or strain. Examples of the former type are structured light and fringe projection, ${ }^{1}$ laser triangulation, ${ }^{2}$ and interferometric distance measurement. ${ }^{3}$ These methods are appropriate where there is sufficient space surrounding the test structure for mounting external light sources and detectors.

A. Fender (a.fender@hw.ac.uk), E. J. Rigg, R. R. J. Maier, W. N. MacPherson, J. S. Barton, A. J. Moore, and J. D. C. Jones are with the Applied Optics and Photonics Group, Physics, School of Engineering and Physical Sciences, Heriot-Watt University, Edinburgh, EH14 4AS, UK. D. Zhao, L. Zhang, and I. Bennion are with the Photonics Research Group, Electronic Engineering and Computer Science Division, Aston University, Birmingham B4 7ET, UK. S. McCulloch and B. J. S. Jones are with the Atomic Weapons Establishment, Aldermaston, Reading RG7 4 PR, UK.

Received 10 July 2006; accepted 11 August 2006; posted 30 August 2006 (Doc. ID 72831).

$0003-6935 / 06 / 369041-08 \$ 15.00 / 0$

(C) 2006 Optical Society of America
Sensors based on optical fibers are the only possible technique for applications such as monitoring aircraft wing surfaces, where a lightweight and compact sensor is required, or guiding microsurgical instruments, where the sensor must also be minimally intrusive. Djordjevich et al. ${ }^{4}$ describe a polymer fiber curvature sensor in which short sections of fiber have part of the cladding and core removed to leave a D-shaped cross section that is susceptible to a large bend loss in the plane of symmetry. They emphasize the advantage of an embedded fiber curvature sensor over conventional strain sensors for measuring the curvature of thin shells, in which the surface strains are small. In addition, the forces required to bend a fiber laterally are small, especially if compared with those required to stress a fiber axially, so provided the fiber is embedded or is in good surface contact with the test structure, it will register the local shape accurately without interfering to a major extent with the object. Fiber Bragg gratings (FBGs) are alternatives to intensity-based bend loss measurement, as they respond to the local strain within a curved fiber. The advantages of FBGs include wavelength-based measurement, interrogation in reflection rather than transmission, ease of multiplexing, and the availability of commercial interrogators with picometer wavelength resolution and accuracy. However, a simple FBG in conventional fiber will not function as an efficient curvature sensor, as the single fiber core is 
concentric with the neutral axis, so the FBG will not experience significant net strain on bending. A FBG bonded at a slant onto the lateral surface of a cantilever beam has been shown to develop a chirp in response to the strain gradient, with consequent spectral broadening proportional to curvature. ${ }^{5}$ The transmitted power in the side modes of a blazed FBG depends on fiber curvature and bend orientation relative to the plane of the blaze angle. ${ }^{6}$

A number of authors have described curvature measurement using fibers with cores offset from the geometrical center. In this case, a grating will not be located on the neutral axis and will therefore experience strain when the fiber is bent. In the simple case of a uniform elastic material such as silica, the strain is linearly related to the radial distance from the neutral axis and is a maximum in the plane of the bend. The sign of the strain changes between the inner and outer sides of the bend. Examples of this approach include FBGs (Ref. 7) and long period gratings ${ }^{8}$ (LPGs) in D-shaped crosssection fibers, LPGs in eccentric core fiber, ${ }^{9}$ a threefiber sensing head, ${ }^{10}$ and FBGs in multicore fiber. ${ }^{11}$ The particular advantages of gratings written in multicore fibers (MCF) compared with single core fibers are the compact size, the ability to measure curvature in two dimensions with one sensing fiber, and the proximity of the cores within a single cladding ensuring good common mode rejection of temperature or longitudinal strain effects on the separate FBGs.

FBG interrogation techniques commonly impose a trade-off between wavelength resolution and measurement time. In order to monitor structural curvature dynamically in real time the required measurement bandwidth may exceed that available from wavelength scanning detection or tunable lasers. If it can be assumed that no external variations of intensity on the time scale of the dynamic event are present, wavelength to intensity measurements can be used. In this paper we present two-dimensional dynamic curvature measurements using arrayed waveguide gratings for ratiometric detection of wavelength shifts of FBGs in MCF.

\section{Arrayed Waveguide Grating Interrogation of Fiber Bragg Gratings}

The nature of the FBG encodes the measurand in the wavelength domain. High bandwidth recovery of the grating wavelength (and hence the measurand) can be effected by using a suitable edge filter to transfer from the wavelength to the intensity domain. Sensitivity to intensity noise (e.g., bend loss) can be reduced by using two filters such that the ratio of the resulting intensity signals is related to the measurand.

Commercial arrayed waveguide gratings (AWG) are available as wavelength division multiplexing components and offer a convenient source of welldefined bandpass filters. By configuring the FBG spectrum to lie between two AWG channels, for example by careful control of the FBG fabrication, it is possible to use the ratio of the power in these chan-

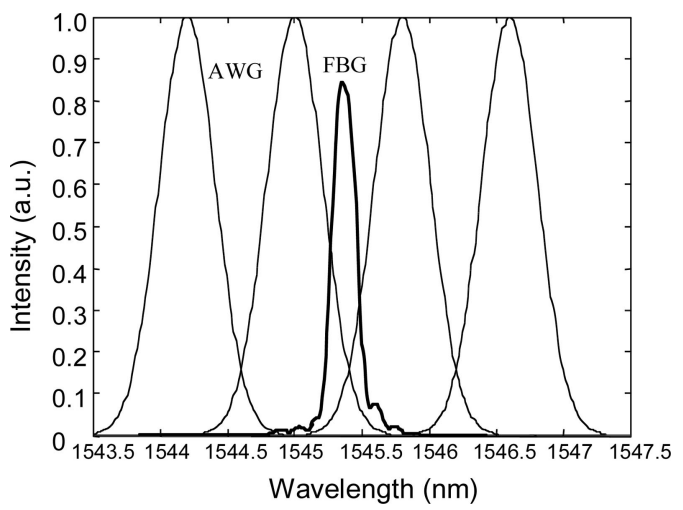

Fig. 1. Modeled spectra of 4 AWG channels and 1 FBG strain sensor.

nels to infer the grating wavelength. According to Sano and Yoshino ${ }^{12}$ in the ideal case of identical AWG and FBG spectral profiles for small FBG wavelength shifts the logarithmic ratio $\rho$ linearizes the conversion to wavelength, i.e.,

$$
\rho_{i}\left(\lambda_{B}\right)=\ln \frac{I_{i+1}}{I_{i}}
$$

where $I_{i}$ is the intensity signal from the $i^{\text {th }}$ channel and $\lambda_{B}$ is the center wavelength of the sensor.

To investigate the expected performance of such a system, AWG channels were modeled as Gaussian spectral profiles with $800 \mathrm{pm}$ spacing and FWHM of $500 \mathrm{pm}$ (Fig. 1). The power transmitted by each channel was calculated using the reflection spectrum of a FBG used later in this work. From this model we expected a strain resolution of better than $1 \mu \varepsilon$ over a $400 \mu \varepsilon$ range with a $200 \mathrm{pm}$ width sensor grating, provided the measured intensity $I_{i}$ is digitized with a ten-bit accuracy. The ratio calculated by applying Eq. (1) to this modeled data suggests that the response is near linear for wavelength deviations $< \pm 150 \mathrm{pm}$ from the midpoint between the two AWG channels (Fig. 2). However, provided this curve is repeatable in practice, it is possible to compensate for any system-

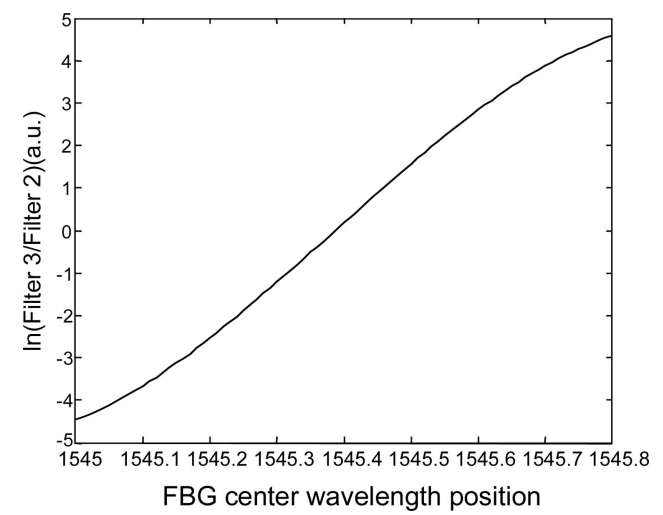

Fig. 2. Logarithmic ratio (channel 3/channel 2) as a function of FBG center wavelength. 


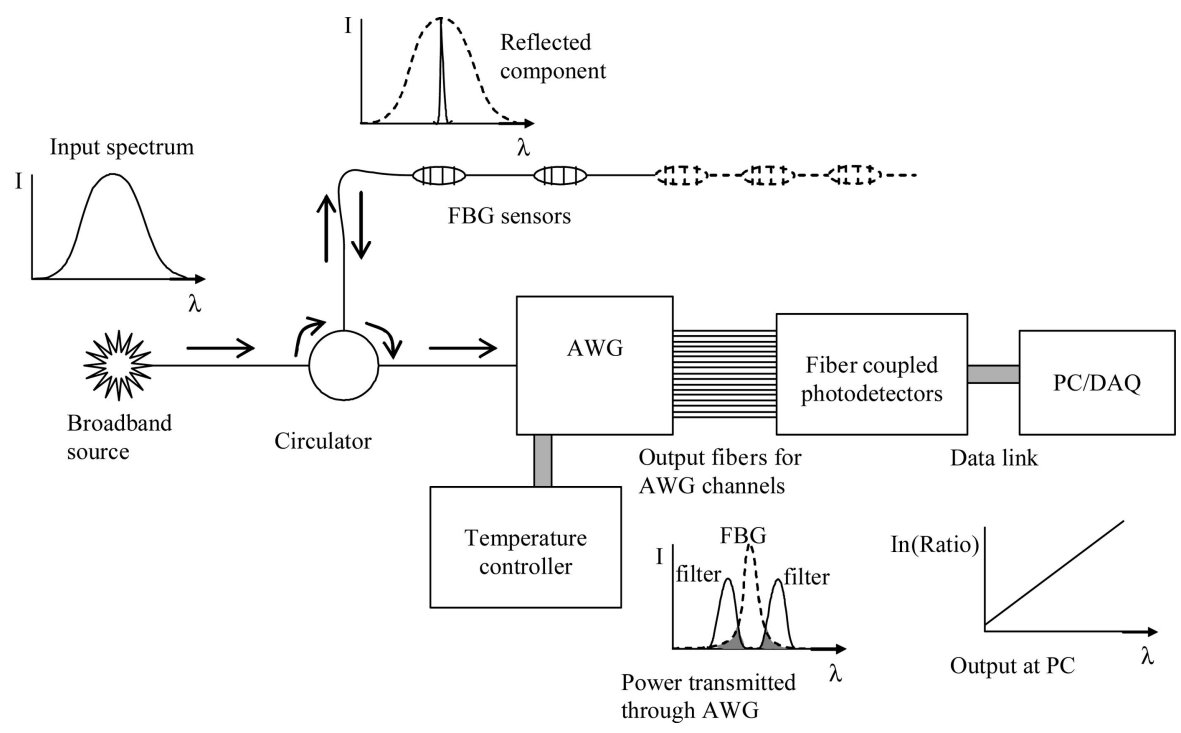

Fig. 3. Schematic arrangement of optical system.

atic nonlinearities, by polynomial or spline fitting, for example.

\section{Calibration and Dynamic Strain Measurement Using an Arrayed Waveguide Grating}

Here we describe the AWG interrogation technique applied to a grating in a conventional single core fiber, and we defer the application to multicore fiber to Section 4. A schematic of the system is shown in Fig. 3. A broadband source illuminated the sensing fiber via a circulator whose output port is connected to the AWG. The relevant AWG output channels were coupled to photodetectors, and logged by a PC data acquisition card for processing and display.

The AWG (Alcatel A1936AWG) had 32 channels at $100 \mathrm{GHz}(800 \mathrm{pm})$ spacing with a Gaussian transmission profile, spanning the range from 1535.8 to $1560.6 \mathrm{~nm}$. The $3 \mathrm{~dB}$ bandwidth of the filter peaks was $\sim 550 \mathrm{pm}$ and the adjacent channel cross talk was approximately $-30 \mathrm{~dB}$. The AWG module was housed in a metal enclosure along with a temperature controller to maintain the module at a constant operating temperature above ambient, typically $\sim 79^{\circ} \mathrm{C}$, to stabilize the channel passbands.

The interrogation system was evaluated by using FBGs in conventional SMF-28 fiber as strain sensors in two configurations: strain measurement on the side of a cantilever for quasi-static and low frequency dynamic testing, and application of strain to a FBG attached to a piezo-electric actuator for kilohertz dynamic testing.

The cantilever consisted of a steel beam of dimensions $20 \mathrm{~mm} \times 3 \mathrm{~mm} \times 200 \mathrm{~mm}$ and unsupported length $150 \mathrm{~mm}$ clamped to a support firmly attached to a steel baseplate. A manual micrometer was used to deflect the cantilever and a linear variable differential transformer (LVDT) measured the displacement $15 \mathrm{~mm}$ from the free end. A FBG was epoxied into a $250 \mu \mathrm{m}$ wide slot on the cantilever surface $10 \mathrm{~mm}$ from the clamping point. During the attach-

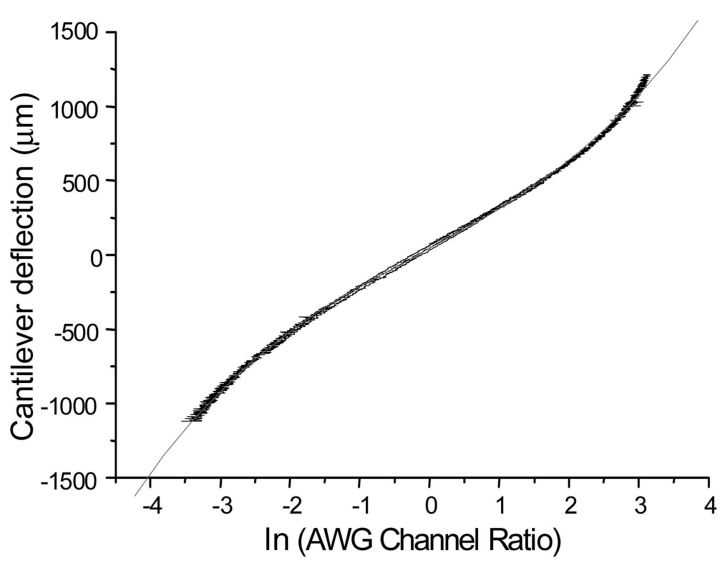

(a)

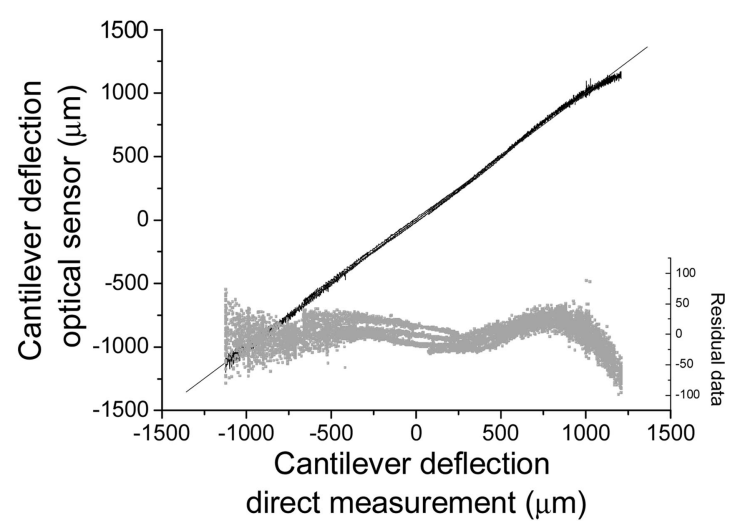

(b)

Fig. 4. (a) Cantilever deflection against log AWG channel ratio, with third-order polynomial fit. (b) Deflection derived from third-order fit versus deflection measured by LVDT. Residuals are shown in the lower plot. 


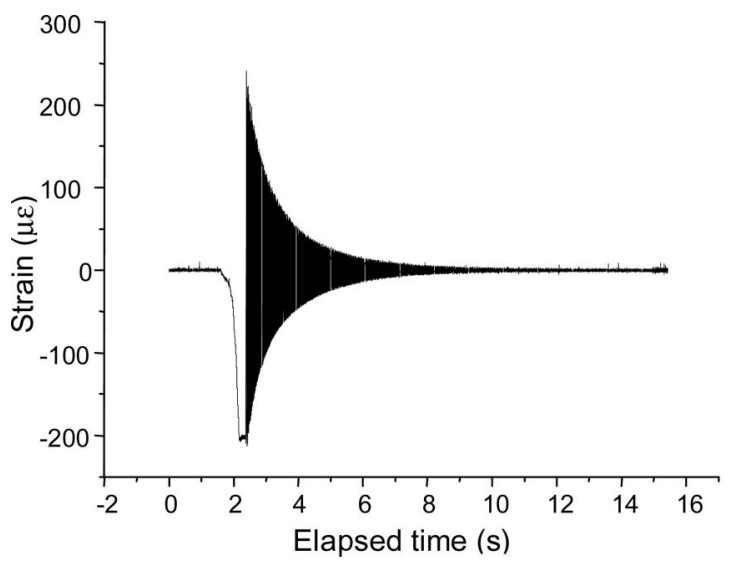

(a)

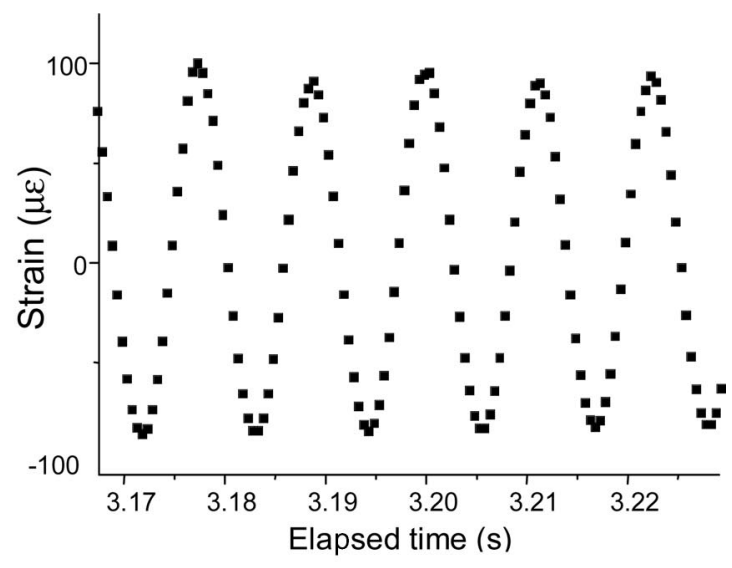

(b)

Fig. 5. (a) Dynamic response of FBG on freely vibrating cantilever. (b) FBG vibration data on expanded time scale, sampling rate $2 \mathrm{kHz}$.

ment process the fiber containing the FBG was held under $\sim 1 \mathrm{~N}$ tension while the epoxy adhesive cured, to apply suitable prestrain to the FBG to ensure that the grating's central wavelength $(1536.4 \mathrm{~nm})$ fell midway between two AWG channels at the nominal AWG operating temperature. The sensor fiber was fusion spliced into the optical system shown in Fig. 3.

Initially the FBG was subjected to strain induced by deflecting the cantilever with the micrometer, monitoring the displacement by the LVDT. The calculation using beam theory ${ }^{13}$ indicated an expected strain of $\sim 200 \mu \varepsilon$ at the sensor for a $1 \mathrm{~mm}$ deflection at the micrometer. Intensity data from the appropriate AWG channels plus the LVDT position monitoring output were captured sequentially at $500 \mathrm{~Hz}$. Data points were taken for deflections in both directions up to a maximum of $\sim 1 \mathrm{~mm}$. The deflection measured by the LVDT is plotted against the logarithmic ratio of adjacent AWG channel signals in Fig. 4(a) according to Eq. (1).

The AWG channel ratio departs markedly from a linear function of deflection over the range $\pm 1 \mathrm{~mm}$. The nonlinear response is primarily due to mismatches in the spectral profiles of the filter and sensor bandpass functions and partially due to background reflectance from Rayleigh scattering and splice return

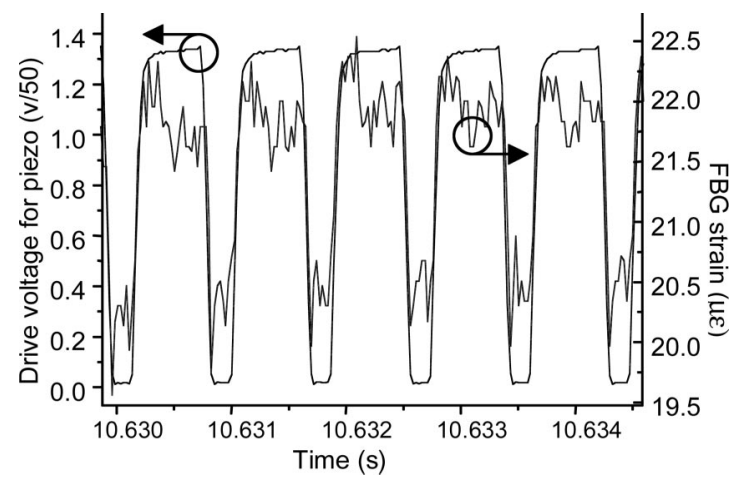

Fig. 6. Strain measurement on piezoelectric cylinder surface driven at $1.1 \mathrm{kHz}$. losses, inevitable in a real system, adding to the AWG channel signals. A third-order polynomial fit linearized the response [Fig. 4(b)] with a standard deviation of $19.7 \mu \mathrm{m}$ in cantilever deflection, equivalent to a strain resolution of $4.2 \mu \varepsilon$.

A dynamic test was carried out by deflecting the cantilever and releasing it, allowing it to ringdown at its natural frequency of vibration of $89 \mathrm{~Hz}$. The resulting strain data, sampled at $2 \mathrm{kHz}$ and linearized using the third-order fit, are shown in Figs. 5(a) and $5(b)$. The measured strain is symmetrical about the mean value.

To test the frequency response in the kilohertz regime, a FBG was attached to the surface of a piezoelectric cylinder. The FBG was applied under a slight prestrain to ensure reliable contact with the cylinder surface and five turns of fiber were wound around the cylinder on either side of the FBGs position. A layer of double-sided tape was used to ensure good adhesion between the cylinder and the fiber.

A standard signal generator excited the piezoelectric cylinder via a high voltage amplifier connected to electrodes on the inner and outer surfaces. The amplifier was capable of driving the cylinder at up to 2.5 $\mathrm{kHz}$ with sufficient amplitude to apply a measurable strain $(\sim 1.5 \mu \varepsilon)$ to the fiber.

Two AWG channels were monitored to interrogate the FBG sensor that was prestrained so that its cen-

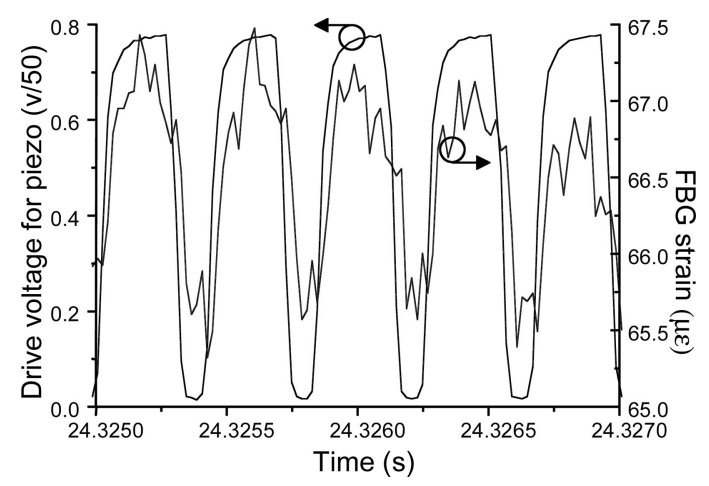

Fig. 7. Same as Fig. 6 for a drive frequency of $2.4 \mathrm{kHz}$. 


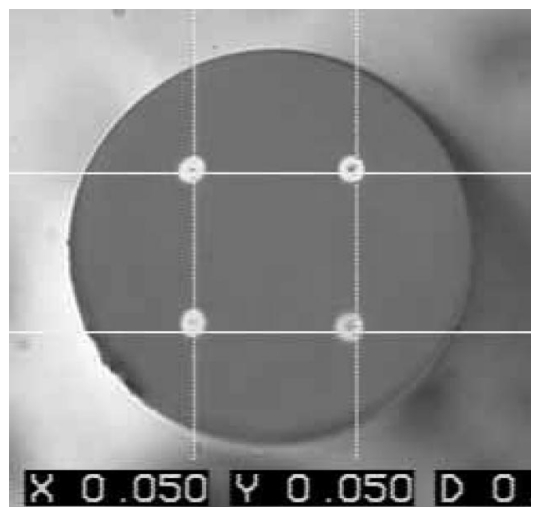

Fig. 8. Cross section of MCF.

tral wavelength fell halfway between the channels. Both AWG channels and the drive voltage were sampled at a rate of $40 \mathrm{kHz}$. The FBG data were converted to strain using the polynomial fits, and the responses to $1.1 \mathrm{kHz}$ and $2.4 \mathrm{kHz}$ vibrations are shown in Figs. 6 and 7.

At $1.1 \mathrm{kHz}$ the sensor shows good response characteristics with $\sim 0.5 \mu \varepsilon$ noise and good repeatability. At $2.4 \mathrm{kHz}$ the noise level is similar, with some evidence of a phase lag between the drive signal and the response, which is likely to be mechanical in origin.

These results show that AWG interrogation holds promise for the high-speed dynamic strain measurement. However, for absolute measurement it is necessary to compensate for measurand cross sensitivities, such as temperature. By using the architecture described here it would be possible to use a reference grating to compensate for thermal effects; alternatively, a differential measurement-afforded by placing a FBG strain sensor on either side of the cantilever-offers a means of eliminating common mode thermal effects. However, this approach fails if there is any inconsistency of strain transfer from the cantilever to the FBG, or if there is a thermal gradient across the cantilever.

MCF offers an alternative means of structural shape monitoring. By measuring curvature of the fiber rather than structural strain the strain transfer requirement is relaxed, ${ }^{4}$ such that it is only important that the fiber accurately follows the shape of

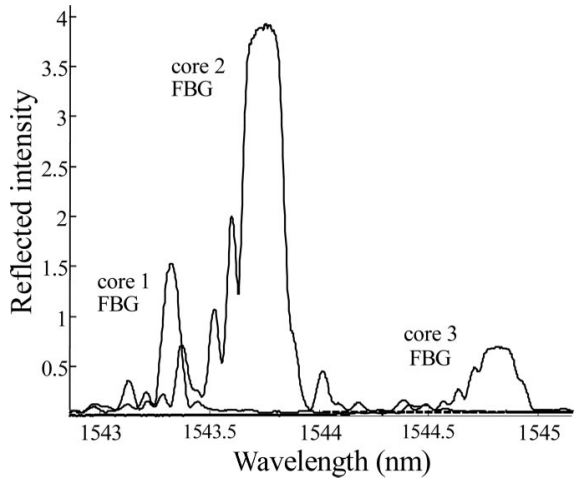

Fig. 9. Reflection spectra of gratings in MCF.

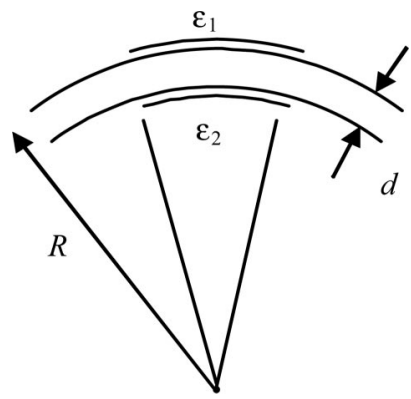

Fig. 10. Differential curvature measurement using two cores of MCF.

the test structure. Furthermore, by incorporating all strain sensors in a single homogeneous fiber it is relatively straightforward to ensure that the fiber is isothermal. For example, in cases of extreme thermal gradients across the test structure thermal barrier coatings can be more easily applied to a thin fiber than a larger sensor. Therefore high bandwidth AWG interrogation of FBG in MCF is potentially attractive for high-speed structural monitoring, while relaxing the attachment and thermal restrictions. Furthermore, because of design of the fiber, two-axis curvature measurement is possible, as described in Section 4.

\section{Dynamic Two-Axis Curvature Measurement Using Multicore Fibers}

In this section, a method for two-axis curvature measurement using AWG interrogation of FBGs at kilohertz frequencies is described. This scheme employs three AWGs to interrogate three cores of a $\mathrm{MCF}$, each containing a FBG.

The multicore fiber has four cores located at the vertices of a $50 \mu \mathrm{m}$ square, as shown in Fig. 8. This fiber was fabricated by inserting four B-Ge-doped silica rods into holes drilled into a silica preform before the fiber was drawn. Each core was $9 \mu \mathrm{m}$ in diameter and was single mode at $1550 \mathrm{~nm}$. The cladding diameter of the fiber was $125 \mu \mathrm{m}$, designed to match that of a standard single-mode fiber, allowing the MCF to be cleaved using a standard cleaver, although splicing requires a fusion splicer capable of rotation alignment (e.g., Vytran ffs-2000). The Vytran splicer has the capacity to view the end face of the fiber and control the fiber orientation. We have used this to produce splices with losses $<1 \mathrm{~dB}$, while maintaining high isolation between cores.

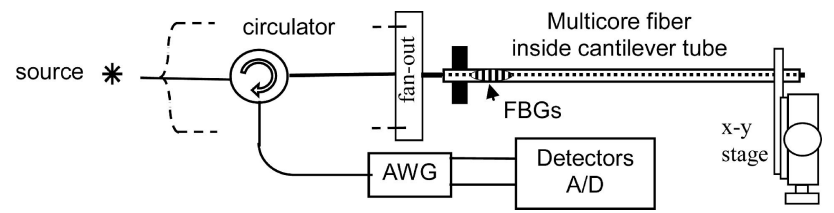

Fig. 11. AWG interrogation of multicore FBGs embedded in a simple cantilever. FBG is located close to the cantilever support and for simplicity, the interrogation scheme is shown for only one of the four cores. 


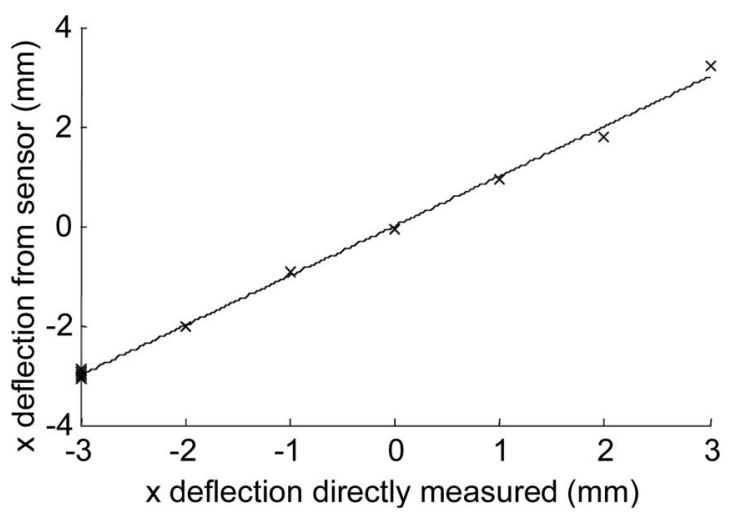

(a)

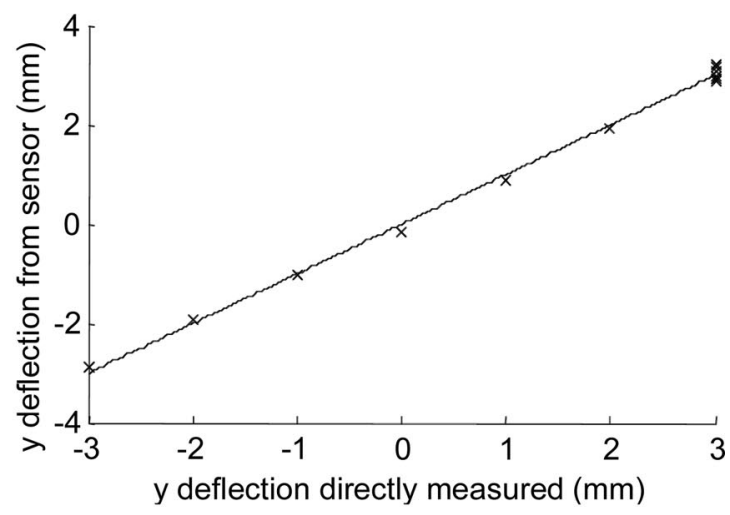

(b)

Fig. 12. (a) $x$ deflection of cantilever measured using the sensor plotted against the directly measured deflection in $x$. (b) $y$ deflection of cantilever measured using the sensor plotted against the directly measured deflection in $y$.

Light may be coupled into or out of each core independently using a specially fabricated fan-out. ${ }^{11}$ The fan-out consisted of four conventional single core fibers (SMF-28) with their diameters reduced to $50 \mu \mathrm{m}$ by etching for $\sim 45 \mathrm{~min}$ in $40 \%$ hydrofluoric acid. The etched fibers were stacked inside a square crosssection capillary such that their cores aligned with the MCF cores, thereby offering a means of coupling the MCF to conventional optical fibers. Typically the coupling loss was $<1 \mathrm{~dB}$ with $>40 \mathrm{~dB}$ isolation between cores.

Gratings were written into three of the MCF cores at the same position along the length of the fiber using a phase-mask scanning technique with a frequency-doubled Ar laser at $244 \mathrm{~nm}$. The MCF gratings used in these tests have a reflection spectra with FWHM $\sim 0.25 \mathrm{~nm}$ and a center wavelength in the region 1543.5-1545 nm, as shown in Fig. 9. These gratings were $\sim 1 \mathrm{~cm}$ in length.

The fiber curvature was obtained from the strains $\varepsilon_{1}$ and $\varepsilon_{2}$ in two cores of the MCF as shown in Fig. 10. The radius of curvature $R$ in the plane of the cores is simply related to the strain difference between gratings 1 and 2 by $^{11}$

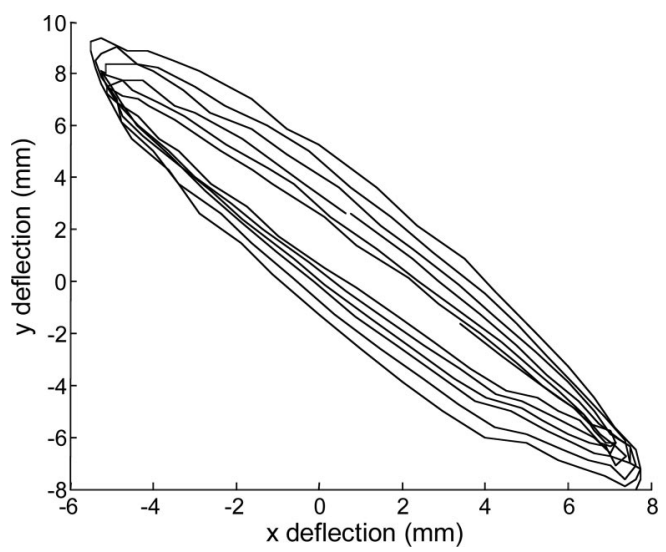

Fig. 13. Plane of cantilever vibration from high-speed camera image data.

$$
R=\frac{d}{\varepsilon_{1}-\varepsilon_{2}}
$$

where $d$ is the spacing between the cores and $\varepsilon_{n}$ is the strain in the $n^{\text {th }}$ core. By using two orthogonal pairs of cores it is possible to obtain curvature in two dimensions.

\section{A. Quasi-Static Tests}

To test the MCF-AWG curvature measurement, the MCF containing the FBGs was placed inside a close fitting stainless steel tube (internal diameter $279 \mu \mathrm{m}$, length $16 \mathrm{~mm}$ ), which was clamped at one end to form a cantilever. The fiber was positioned such that the gratings were located close to the clamp, where the curvature was greatest. Figure 11 illustrates the experimental setup.

To remove any constant offset in the AWG channel intensities arising from unwanted reflections, for example, background values were measured by attenuating the signal via small loops in the MCF between the fan-out and the cantilever.
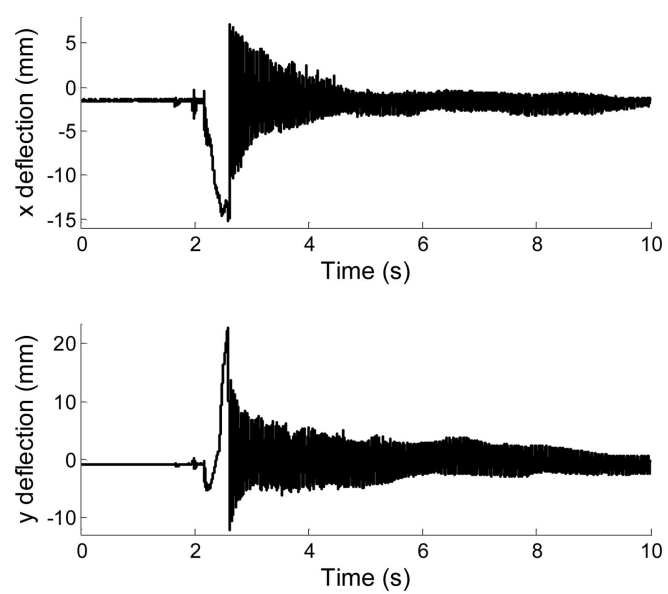

Fig. 14. $x$ and $y$ dynamic ringdown of MCF FBGs inside cantilever tube. 


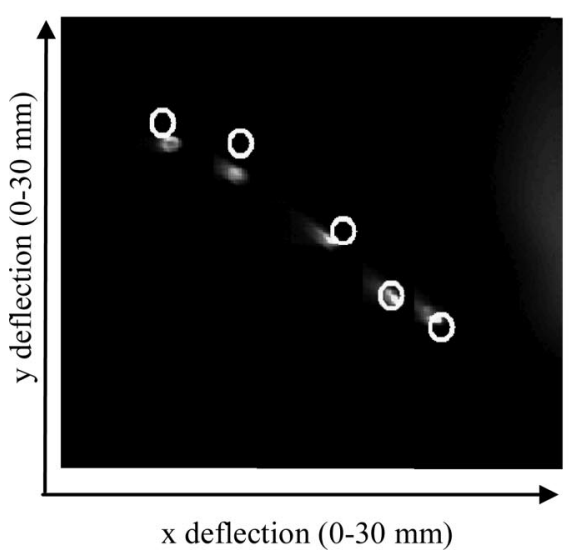

(a)
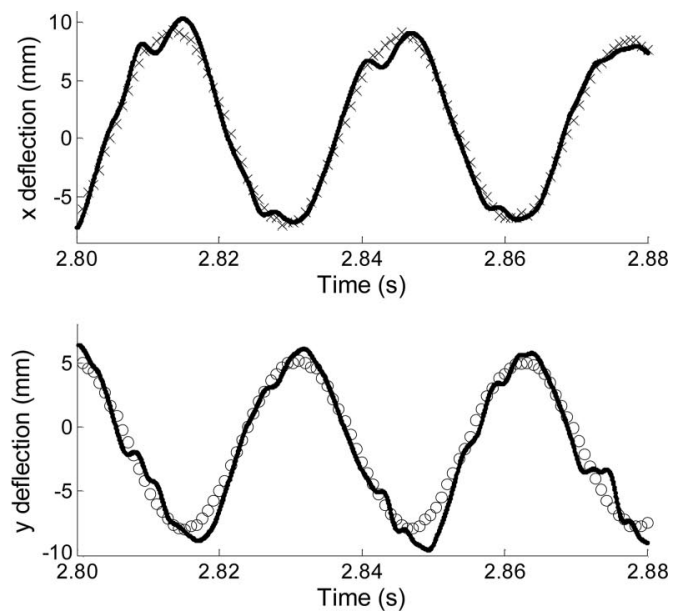

(b)

Fig. 15. (a) Sequence of images from high-speed camera at $4 \mathrm{~ms}$ intervals. Circles show corresponding sensor measurement. (b) Upper plot, $x$ deflection measured by MCF FBGs (solid curve) and camera (x). Lower plot, $y$ deflection measured by sensor (solid curve) and camera $(\bigcirc)$.

An $x-y$ stage was used to deflect the cantilever through a set of displacements equivalent to a $6 \times$ $6 \mathrm{~mm}$ grid at $1 \mathrm{~mm}$ intervals. The orientation of the fiber axes relative to the $x$ and $y$ directions was arbitrary. At each grid position, the $x$ and $y$ strain values, calculated from the output voltages of the sensing AWGs, were recorded. The matrix for recovering the $x$ and $y$ position values from the strain measurements was found by fitting a plane equation to each set of strain measurements:

$$
\left[\begin{array}{l}
\varepsilon_{1} \\
\varepsilon_{2}
\end{array}\right]=\left[\begin{array}{lll}
c_{1} & c_{2} & c_{3} \\
c_{4} & c_{5} & c_{6}
\end{array}\right]\left[\begin{array}{l}
x \\
y \\
1
\end{array}\right]
$$

where $c_{1}$ to $c_{6}$ are constants.

Figure 12 shows the cantilever deflection reconstructed from the FBG measurements plotted against the directly measured cantilever deflection over the $6 \mathrm{~mm}$ range. The standard deviation of a linear fit to the response is $0.4 \mathrm{~mm}$ for both $x$ and $y$ axes. This indicates a curvature resolution of $0.05 \mathrm{~m}^{-1}$, which is equivalent to a maximum detectable bend radius of $20 \mathrm{~m}$.

\section{B. Dynamic Tests}

To demonstrate dynamic two-axis curvature measurement, the $x-y$ stage was removed and a highspeed camera (Kodak 4540) was used to image the displacement of the end of the cantilever during cantilever oscillation. Using appropriate calibration data enabled a direct comparison between the camera displacement measurements and the cantilever displacement predicted by the FBG curvature measurement.

The plane of the cantilever vibration was arbitrary and the cantilever end position measured by the camera is shown in Fig. 13. The camera frame rate was 1125 frames per second, while $x$ and $y$ strain data were recorded at 11,250 $\mathrm{Hz}$. The experimental data were downsampled using ten-point averaging to give an effective sampling rate of $1125 \mathrm{~Hz}$, equivalent to the camera frame rate.

The measured ringdown of the cantilever vibration for the $x$ and $y$ directions is shown in Fig. 14. The frequency of this vibration, from these results, was found to be $28 \mathrm{~Hz}$. This compares well with the lowest natural frequency of $30 \mathrm{~Hz}$ predicted from the properties of the cantilever tube.

Selected images from the camera, at $4 \mathrm{~ms}$ intervals, are shown in Fig. 15(a), along with the corresponding displacement measurement from the sensor. The same results are shown in Fig. 15(b) with displacement from the camera and the sensor plotted on the same axes. The standard deviation of the difference between the camera and sensor displacements was $0.9 \mathrm{~mm}$ for both $x$ and $y$ axes. This indicates a curvature resolution of $0.09 \mathrm{~m}^{-1}$, or a maximum bend radius of $11 \mathrm{~m}$ measured at $1125 \mathrm{~Hz}$.

\section{Conclusions}

We have shown that the differential strain between orthogonal pairs of Bragg gratings in multicore fiber can resolve fiber curvature to $0.05 \mathrm{~m}^{-1}$ in two axes simultaneously. The multicore fiber grating technique enables the curvature to be measured by a sensing element no larger than a conventional silica fiber, and therefore is applicable in circumstances in which limited access prevents the use of full field or imaging methods. Arrayed waveguide gratings can be used to convert Bragg wavelength shifts to intensity changes, permitting curvature to be measured dynamically at kilohertz frequencies. The technique has been demonstrated by tracking an arbitrary twodimensional cantilever oscillation at $28 \mathrm{~Hz}$.

The authors acknowledge the support of the UK Engineering and Physical Science Research Council (EPSRC) and AWE plc. W. MacPherson thanks the 
EPSRC for funding via the Advanced Fellowship Scheme. The authors also thank Gary Fleming, NASA Langley, for helpful discussions and provision of the multicore fiber.

\section{References}

1. C. J. Tay, M. Thakur, and C. Quan, "Grating projection system for surface contour measurement," Appl. Opt. 44, 1393-1400 (2005)

2. V. A. Sujan and S. Dubowsky, "Design and implementation of a 3-D mapping system for highly irregular shaped objects with application to semiconductor manufacturing," Opt. Eng. 41, 1406-1417 (2002).

3. P. A. Coe, D. F. Howell, and R. B. Nickerson, "Frequency scanning interferometry in ATLAS: remote, multiple, simultaneous and precise distance measurements in a hostile environment," Meas. Sci. Technol. 15, 2175-2187 (2004).

4. A. Djordjevich, M. Fung, and R. Y. K. Fung, "Principles of deflection-curvature measurement," Meas. Sci. Technol. 12, 1983-1989 (2001).

5. X. Dong, H. Meng, Z. Lui, G. Gai, and X. Dong, "Bend measurement with chirp of fiber Bragg grating," Smart Mater. Struct. 10, 1111-1113 (2001).

6. S. Baek, Y. Jeong, and B. Lee, "Characteristics of short-period blazed fiber Bragg gratings for use as macro-bending sensors," Appl. Opt. 41, 631-636 (2002).
7. F. M. Araújo, L. A. Ferreira, J. L. Santos, and F. Farahi, "Temperature and strain insensitive bending measurements with D-type fiber Bragg gratings," Meas. Sci. Technol. 12, 829 833 (2001)

8. D. H. Zhao, X. F. Chen, K. M. Zhou, L. Zhang, I. Bennion, W. N. MacPherson, J. S. Barton, and J. D. C. Jones, "Bend sensors with direction recognition based on long-period gratings written in D-shaped fiber," Appl. Opt. 43, 5425-5428 (2004).

9. H. Patrick, "Self-aligning, bipolar bend transducer based on long period grating written in eccentric core fiber," Electron. Lett. 36, 1763-1764 (2000).

10. F. M. Araújo, L.A. Ferreira, and J. L. Santos, "Simultaneous determination of curvature, plane of curvature, and temperature by use of a miniaturized sensing head based on fiber Bragg gratings," Appl. Opt. 41, 2401-2407 (2002).

11. G. M. H. Flockhart, W. N. MacPherson, J. S. Barton, J. D. C. Jones, L. Zhang, and I. Bennion, "Two-axis bend measurement with Bragg gratings in multicore optical fiber," Opt. Lett. 28, 387-389 (2003).

12. Y. Sano and T. Yoshino, "Fast optical wavelength interrogator employing arrayed waveguide grating for distributed fiber Bragg grating sensors," J. Lightwave Technol. 21, 132-139 (2003).

13. J. M. Gere and S. P. Timoshenko, Mechanics of Materials (PWS, 1997) 